\title{
Consumo de pornografía y su impacto en actitudes y conductas en estudiantes universitarios ecuatorianos
}

\author{
Marie-France Merlyn ${ }^{1}$, Liliana Jayo², Doris Ortiz ${ }^{3}$ y Rodrigo Moreta-Herrera ${ }^{4}$
}

\begin{abstract}
RESUMEN
Las nuevas tecnologías de la información y comunicación han permitido el acceso sin ningún filtro a contenidos sobre sexualidad en la red, sobre todo a pornografia, la que se constituye en una fuente de información y cuyo consumo acarrea consecuencias tanto en lo individual como en lo relacional. En este marco, el objetivo del presente estudio es recabar información en la población de 18 a 25 años sobre pornografia en general, exposición, uso actual y sus posibles consecuencias en las actitudes y conductas sexuales. El estudio se realizó en dos fases: en la primera, de índole cualitativa, se realizaron 54 entrevistas grupales semiestructuradas con 122 mujeres y 120 hombres; en la segunda, cuantitativa, 590 jóvenes llenaron una encuesta en línea $(56.4 \%$ mujeres; $43.6 \%$ hombres). Los resultados de las entrevistas ligan la pornografia a temas de facilidad de Acceso, Incitación a niños y adolescentes a un despertar temprano de la curiosidad sexual y Afectación, sobre todo a nivel de sexualidad. Los resultados de la encuesta muestran que el $78 \%$ de los jóvenes ha estado expuesto a pornografia por lo menos una vez en su vida, que por lo general fue en la adolescencia temprana, y que existe un consumo actual en $44.58 \%$ de la muestra. Además, los resultados arrojan un impacto del ver pornografia en las actitudes hacia la pornografia, las relaciones sexuales y el placer. Las consecuencias se presentan también en las conductas sexuales que tienen los usuarios de pornografia con su pareja durante una relación sexual, ya que reportan haber realizado algunas conductas violentas sin autorización de la misma de manera significativamente superior a las personas que no ven pornografia. Se concluye que la pornografia tiene consecuencias negativas en la sexualidad, y sobre la importancia de la educación sexual para contrarrestar la información negativa que vehicula la pornografia.
\end{abstract}

Palabras Clave: pornografia, juventud, impacto, conductas, actitudes.

\section{Pornography consumption and its impact on attitudes and behaviors in ecuadorian university students}

\begin{abstract}
The new information and communication technologies have allowed access without any filter to sexuality content on the Internet, especially pornography, which constitutes a source of information and whose consumption has consequences both individually and relationally. Within this framework, the objective of the present study was to collect information in the population aged 18 to 25 on pornography in general, exposure, current use and its possible consequences on sexual attitudes and behaviors. The study was
\end{abstract}

\footnotetext{
1 Pontificia Universidad Católica del Ecuador, Ecuador; mfmerlyns@puce.edu.ec; https://orcid.org/00000001-6171-2691

2 Pontificia Universidad Católica del Ecuador, Ecuador; ljayo@puce.edu.ec; https://orcid.org/0000-00020080-4020

${ }^{3}$ Pontificia Universidad Católica del Ecuador, Ecuador; dortiz107@puce.ec; https://orcid.org/0000-00030617-0361

4 Pontificia Universidad Católica del Ecuador, Ecuador; rmoreta@pucesa.edu.ec; https://orcid.org/00000003-0134-5927
} 
carried out in two phases: in the first, of a qualitative nature, 82 semi-structured group interviews were carried out with 122 women and 120 men; in the second, quantitative, 590 young people filled out an online survey (56.4\% female; $43.6 \%$ male). The results of the interviews link pornography to issues of easy Access, Incitement of children and adolescents to an early awakening of sexual curiosity and Affectation, especially in sexuality. The survey results show that $78 \%$ of young people have been exposed to pornography at least once in their life, generally during early adolescence, and that there is a current consumption at $44.58 \%$ of the sample. In addition, the results show an impact of seeing pornography on attitudes towards pornography, sexual relations and pleasure. The consequences also occur in the sexual behaviors that porn users have with their partner during a sexual relationship, since they report having performed some violent behaviors without authorization from it in a significantly superior way to people who do not see pornography. It is concluded that pornography has negative consequences on sexuality, and on the importance of being able to counteract the information that it conveys with formal sexual education.

Keywords: pornography, youth, impact, behavior, attitudes.

La explosión y crecimiento de las nuevas tecnologias de la información y la comunicación, generados a partir de los años ochenta, trajeron consecuencias innegables para la cotidianidad de los seres humanos. Estos incorporaron rápidamente en su vida diaria a las máquinas, tales como computadoras y portátiles, y con el surgimiento de internet abrieron la puerta de todo lo que circula por él (tanto positivo como negativo) en varios ámbitos, como son: entretenimiento, información, noticias, trabajo, educación y obviamente asuntos más personales y privados como lo sexual. En este último campo, el uso de la red con fines sexuales es ampliamente documentado y estudiado (Castro-Calvo, Ballester-Arnal, Giménez-García, \& Gil-Juliá, 2017).

Si bien lo que existe en la red como información a nivel sexual es variado, mucho del contenido propuesto es material explícito, es decir pornografia. Con el surgimiento de las nuevas tecnologias, esta pasó de ser algo dificil de conseguir, ilegal y usado por individuos de dudosa moralidad, a ser un nuevo producto: existe una clara diferencia entre la pornografia convencional (impresa o digital), cuyo impacto era limitado justamente por el costo elevado de producción, una distribución difícil y la alta exposición del consumidor para obtenerla, con esta "nueva pornografia", de mejor calidad, accesible, sin límite, y que garantiza a su consumidor el anonimato si asî lo desea (Ballester, Orte, \& Pozo, 2019). Más allá, en los últimos años se observa que las parejas también envian sus vídeos de producción "artesanal", lo que muestra que este fenómeno excedió lo que la propia industria produce. La pornografia, según Szil (2018), ha ido "intoxicando a la cultura sexual cotidiana" (p. 128), estableciendo conductas y patrones que le son propios, que se instalan como modas y se normalizan socialmente. Así se pueden observar, por ejemplo, el tener los genitales afeitados, los golpes en las nalgas, la estrangulación y el sexo anal (Szil, 2018) entre otros.

La cultura en el nuevo milenio incorporó la pornografia en la vida cotidiana de las personas y la ha hecho presente en espacios externos como blogs, foros, revistas digitales de sexualidad que proponen técnicas y prácticas sexuales comunes en la pornografia (como el sexo anal); o que aconsejan a las mujeres sobre cómo entender el uso de la pornografia en los hombres, sobre la base de la idea de que en ellos esto es 'biológicamente normal' al ser 'más diversos' y 'visuales' (Szil, 2018). El uso de la pornografia se extendió, y se lo propone como base de la terapia sexual o como fuente de educación sexual 
desde el mundo académico (Farvid \& Braun, 2014; Favaro \& De Miguel, 2016; Tyler, 2011).

En la actualidad, las cifras de búsqueda de pornografia son altas. Así, el $20 \%$ de las búsquedas hechas en teléfonos móviles son sobre este tema (Buchholtz, 2019). A nivel mundial, las visitas a páginas pornográficas se incrementan anualmente. Pornhub, por ejemplo, alcanzó en 2018 los 100 millones de visitas en un día (Pornhub, 2018). En Ecuador, de los 10 sitios web más visitados, las páginas pornográficas http//:xnxx.com y http//:xvideos.com ocupan el $7^{\circ}$ y $8^{\circ}$ lugar respectivamente (Del Alcázar, 2018).

\section{Definición de pornografía}

Pero ¿qué es exactamente la pornografia? Etimológicamente, pornografía proveniente del griego, hace alusión a la representación gráfica de escenas de prostitución (Castellanos, 2006). Es evidente que en la actualidad encarna mucho más, y es por eso que esta acepción consta en tercer lugar en la Real Academia Española (2019), quien en su primer significado propone: "Presentación abierta y cruda del sexo que busca producir excitación", significado más preciso y amplio para describir lo que se entiende ahora por pornografia. La pornografia debe ser diferenciada de otras representaciones sobre la sexualidad como el erotismo, ya que este último se distingue de la pornografia por cuanto su objetivo no es la incitación sexual o la excitación per se, así como por la ausencia de coerción o de violencia en sus representaciones (Castellanos, 2006). Además, la pornografia mainstream enfatiza las desigualdades de poder entre los géneros, reforzando el estatus subordinado del placer femenino al masculino, erotizando muchas veces la violencia (Castellanos, 2006). Lo generalmente representado en la pornografia exalta el poder de los hombres por encima de las mujeres, estableciendo representaciones que cosifican a la mujer y se centran en el deseo sexual masculino, y fijan en el imaginario social ciertas prácticas que no son eróticas, como el dolor físico en las mujeres, la falta de deseo, el sufrimiento, la humillación, los abusos sexuales a menores, el consumo de prostitución (Alario, 2018).

Alineados a esta visión, autores como Szil (2018) consideran que no nos hemos alejado de la definición original de la palabra, y que la pornografía es el primer elemento de un continuo de dominación masculina sobre la mujer en el terreno sexual, en el que se ubican después la prostitución y la violencia. Tyler (2015) señala que no es nada más que una forma de prostitución más barata para el que la consume, y que trae las mismas consecuencias a nivel psicológico para los que se hallan involucrados en ella, prostitutas/actrices y clientes/usuarios.

Más allá de lo que se piense, la pornografia constituye material sexual explícito que transmite información sobre las relaciones sexuales: tipos de relaciones, escenarios y guiones de copulación, potenciales parejas, posturas. También transmite modelos de lo masculino, de lo femenino, ideales sobre el cuerpo, el desempeño sexual, lo deseable, lo permitido, dejando de lado la afectividad de la sexualidad e imponiendo un modelo de lo que 'debería ser' el sexo, a partir de representaciones tradicionales, creando discursos sobre lo 'normal', 'saludable', 'permitido', 'satisfactorio', entre otros (Rodríguez, González, \& Paulini, 2018). 


\section{Consecuencias de la pornografía}

De manera preocupante, tanto por la facilidad de acceso a la información como por la ausencia de un rol activo de padres e instituciones educativas, la pornografia se está constituyendo en la fuente primaria de niños y adolescentes para obtener información sobre la sexualidad; a menudo antes de que estructuren su propia sexualidad (Albury, 2014; Ballester, Orte \& Pozo, 2019; Del Barrio-Álvarez \& Garrosa, 2015; Tallon-Hicks, 2016), sobre todo cuando por asuntos políticos o tabús no se imparte una educación sexual formal, que abarque más allá del aspecto anatómico-biológico (Rojo, 2018). Esto no ocurre sin consecuencias: varios estudios encuentran una relación directa entre observación de pornografia y reproducción de lo visto en población adolescente: los adolescentes copian lo que ven y solicitan explícitamente a sus parejas actos sexuales observados en la pornografia (Rothman \& Adhia, 2015; Rothman, Kaczmarsky, Burke, Jansen, \& Baughman, 2015).

Adicionalmente, el consumo de pornografia se ha relacionado con diversas consecuencias negativas en la sexualidad. A nivel de conductas sexuales, se encuentra que el consumo de pornografia genera baja satisfacción sexual y mayor excitación (Villena \& Chiclana, 2018); mayor incertidumbre sexual y actitudes más positivas para la exploración sexual sin compromiso (Peter \& Valkenburg, 2008); prácticas sexuales poco seguras y mayor número de parejas sexuales (Harkness, Mullan, \& Blaszczynski, 2015); ausencia de uso del preservativo en relaciones casuales (Wright, Miezan, Sun \& Steffen, 2019), prácticas de alto riesgo y posible demanda a parejas para reproducirlas o consumo de prostitución (Ballester, Orte, \& Pozo, 2019) e incluso adicción a la pornografia (Velasco \& Gil, 2017).

En un nivel más interno, se discute sobre el modelamiento del erotismo a través de las representaciones propuestas por la pornografia (Montoya \& Vélez, 2016), ritualización de la sexualidad (Ballester, Orte, \& Pozo, 2019), influencia en las expectativas sobre la relación sexual (Villena \& Chiclana, 2018), afectaciones en la autoimagen como: inseguridad sobre la apariencia fisica (Johnson, Ezzell, Bridges, \& Sun, 2019), y comparación del cuerpo con el de los actores y actrices (Villena \& Chiclana, 2018).

A nivel relacional, se encontraron consecuencias como pérdida de libido en las relaciones de pareja, evitación sexual, ansiedad (Esteban, 2017), y disminución del placer de actos sexuales íntimos como los besos o las caricias durante el acto sexual (Johnson et al., 2019). Otras posibles consecuencias de la exposición a la pornografía implican la modificación de las actitudes y creencias hacia la aceptación de la violencia en la sexualidad. Así, algunos estudios vinculan al consumo de pornografia con estilos sexuales agresivos y violencia (metaanálisis de 46 estudios de Oddone-Paolucci, Genuis \& Violato, 2017; metaanálisis en 7 países de 22 estudios de Wright, Tokunaga \& Kraus, 2016; Del Barrio-Álvarez y Garrosa, 2015; Kjellgren et al, 2010; Ybarra et al., 2011), creencias sexuales adversas, aceptación del mito de la violación y conservadurismo sexual (Emmers-Sommer, 2018), misoginia (Bischmann et al., 2017) e incluso agresión sexual (Tardif, Pascuzzo, \& Costa, 2015).

Al ser evidente la importancia del tema, y ante la ausencia de estudios en Ecuador, cabe preguntarse si el consumo de pornografia está presente en la población, sobre todo en la franja de la juventud, que son las personas que están estableciendo relaciones de pareja. Por lo tanto, el objetivo del presente estudio es recabar información sobre el uso de pornografia en general, su 
exposición y sus posibles consecuencias en las actitudes y conductas sexuales de la población juvenil ecuatoriana.

\section{MÉTODO}

El presente estudio es de tipo descriptivo, con metodología cualicuantitativa. Se realizó en dos fases, la primera de tipo cualitativo, a través de entrevistas grupales semiestructuradas, y la segunda de corte cuantitativo, a través de una encuesta en línea.

\section{Participantes}

Participaron en las entrevistas 122 mujeres y 120 hombres voluntarios de una institución de educación superior de la ciudad de Quito (ver tabla 1). Para la construcción de los grupos de entrevistados se consideraron que las variables que podian influir en la información a obtener eran: la edad (2 grupos: 18-21 años y 22-25 años), el género (masculino-femenino) y la facultad de la que provenian los estudiantes dentro de la institución (14 facultades). La duración promedio de las entrevistas fue de 30 minutos, y la mayoria estuvo conformada por 3 a 5 participantes.

Tabla 1.

Distribución de los participantes en las entrevistas grupales.

\begin{tabular}{lcccc}
\hline & \multicolumn{2}{c}{ Mujeres } & \multicolumn{2}{c}{ Hombres } \\
\cline { 2 - 5 } FACULTADES & $\mathbf{1 8 - 2 1}$ & $\mathbf{2 2 - 2 5}$ & $\mathbf{1 8 - 2 1}$ & $\mathbf{2 2 - 2 5}$ \\
\cline { 2 - 5 } Arquitectura, Diseño y Artes & 3 & 3 & 4 & 5 \\
Ciencias Administrativas y Contables & 5 & 5 & 5 & 3 \\
Ciencias Exactas y Naturales & 4 & 5 & 3 & 5 \\
Ciencias Filosóficas - Teológicas & 3 & 3 & 5 & 7 \\
Ciencias Humanas & 5 & 5 & 5 & 5 \\
Comunicación, Lingüística y literatura & 3 & 5 & 5 & 4 \\
Economía & 4 & 4 & 5 & 5 \\
Enfermería & 5 & 4 & 5 & 5 \\
Ciencias de la Educación & 5 & 5 & 4 & - \\
Psicología & 5 & 5 & 5 & 5 \\
Ingeniería & 4 & 3 & 6 & 3 \\
Jurisprudencia & 10 & 5 & 5 & 3 \\
Medicina & 3 & 3 & 3 & 6 \\
Trabajo Social & 5 & 3 & 4 & - \\
\hline Nota: Los dos valores faltantes corresponden a entrevistas que no se pudieron realizar \\
porque el número de varones matriculados en esas facultades es muy bajo y no se \\
obtuvo voluntarios.
\end{tabular}

En la segunda fase del estudio se aplicó una encuesta en línea a una muestra no probabilística de 590 sujetos voluntarios. Respondieron esta encuesta 56,4\% mujeres (promedio de edad: 20.69 años; D.T.= 2.03) y 43,6\% hombres (promedio de edad: 21.13 años; D.T.=1.99). En cuanto a la orientación sexual, la distribución fue de: $86 \%$ heterosexuales, $13 \%$ bisexuales, y $2 \%$ homosexuales.

\section{Materiales}

Entrevistas grupales. Para la realización de las entrevistas se contó con una guia semiestructurada de preguntas para conducir el grupo, que indagaba sobre: 1) Conceptos generales sobre sexualidad, 2) Conductas sexuales en la época actual, 3) Diferencias de género, 4) Placer, 5) 
Responsabilidad, 6) Relaciones de pareja, y 7) Problemas en la sexualidad. En cuanto al contenido que se analiza aquí (la pornografía), surgió al abordar el tema 2 (Conductas sexuales en la época actual), que contenía tres preguntas: 1) ¿Qué aspectos han cambiado en la sexualidad de los jóvenes en el momento actual?, 2) ¿Cómo han influenciado la tecnología y los medios de comunicación en la sexualidad? y 3) ¿Qué prácticas o comportamientos sexuales están a la moda?

Encuesta. Para la encuesta, los investigadores generaron items sobre la base de los conceptos revisados en la literatura y de la información obtenida en las entrevistas. Estos items sirvieron para recoger información sobre: 1) Acceso a pornografia y consumo actual, 2) Actitudes hacia la pornografia, comportamientos, relaciones sexuales y placer, y 3) Conductas sexuales violentas realizadas sin consentimiento durante el acto sexual.

Preguntas sobre acceso a pornografia y consumo actual. Se elaboraron 6 items para este tema, como se presenta en la tabla 2 a continuación.

Tabla 2.

Construcción de las preguntas sobre acceso a pornografía y consumo actual.

\begin{tabular}{|c|c|c|c|c|}
\hline Tema & $\begin{array}{l}\text { Número } \\
\text { de ítems }\end{array}$ & Subtema & $\begin{array}{l}\text { Opciones de } \\
\text { respuesta }\end{array}$ & Ítem \\
\hline \multirow{3}{*}{$\begin{array}{l}\text { Primer } \\
\text { acceso }\end{array}$} & \multirow[b]{3}{*}{3} & Existencia & Sí /No & ¿Has tenido acceso a la pornografía? \\
\hline & & Edad & Numérica & $\begin{array}{l}\text { ¿A qué edad accediste por primera vez a } \\
\text { contenido pornográfico? }\end{array}$ \\
\hline & & Fuentes & Sí /No & $\begin{array}{l}\text { ¿Desde cuales fuentes accediste a la } \\
\text { pornografía? Opciones: prensa escrita, } \\
\text { internet, redes sociales, televisión por cable, } \\
\text { videos, revistas }\end{array}$ \\
\hline \multirow{3}{*}{$\begin{array}{l}\text { Consumo } \\
\text { actual }\end{array}$} & \multirow{3}{*}{3} & Existencia & Sí /No & Actualmente, ¿ves pornografía? \\
\hline & & $\begin{array}{l}\text { Tiempo de } \\
\text { uso } \\
\text { semanal }\end{array}$ & Numérica & $\begin{array}{l}\text { ¿Cuánto tiempo por semana le dedicas a la } \\
\text { pornografía? }\end{array}$ \\
\hline & & Tipo & Sí /No & $\begin{array}{l}\text { ¿Cuál es el tipo de pornografía que ves? ( } 9 \\
\text { opciones de respuesta) }\end{array}$ \\
\hline
\end{tabular}

Preguntas sobre Actitudes. Un listado de actitudes respecto a pornografia y sexualidad (comportamientos, relaciones sexuales y placer) fue redactado por los investigadores, validado por tres psicólogos expertos en sexualidad, y sometido a un piloto con un grupo de 13 estudiantes. El listado final quedó compuesto por 11 afirmaciones que constituyen actitudes hacia la pornografia, 12 hacia los comportamientos sexuales y 25 hacia las relaciones sexuales y el placer, principalmente creencias erróneas y distorsiones sobre el tema (ver tabla 3). Estas pasaron por un proceso de verificación de su fiabilidad con el coeficiente de Cronbach (a), junto con sus intervalos de confianza al 95\% (IC), para conocer la consistencia interna que guardan los ítems entre sí en función de los factores desarrollados. La pregunta general que se hizo sobre las actitudes fue: ¿En qué medida estás de acuerdo con las siguientes afirmaciones...? Los participantes contestaron a través de una escala Likert de 5 puntos (desde totalmente en desacuerdo a totalmente de acuerdo). 
Tabla 3.

Construcción de las escalas sobre actitudes.

\begin{tabular}{lcccc}
\hline Tema & $\begin{array}{c}\text { Fiabilidad de } \\
\text { los factores }\end{array}$ & Subtema & $\begin{array}{c}\text { Número } \\
\text { de ítems }\end{array}$ & Ejemplos de ítem \\
\hline $\begin{array}{l}\text { Actitudes hacia la } \\
\text { pornografía }\end{array}$ & $\begin{array}{l}.82 \\
{[.80-.84]}\end{array}$ & Actitudes positivas & 9 & $\begin{array}{c}\text { Enseña cosas interesantes sobre las } \\
\text { relaciones sexuales }\end{array}$ \\
\hline $\begin{array}{l}\text { Actitudes hacia los } \\
\text { comportamientos } \\
\text { sexuales }\end{array}$ & $\begin{array}{l}.78 \\
{[.75-.81]}\end{array}$ & - & 2 & $\begin{array}{c}\text { Debería ser objeto de regulaciones } \\
\text { estrictas o incluso prohibida }\end{array}$ \\
\hline $\begin{array}{l}\text { Actitudes hacia las } \\
\text { relaciones } \\
\text { sexuales y placer }\end{array}$ & {$[.80-.84]$} & Relaciones sexuales & 10 & $\begin{array}{c}\text { Es importante que la mujer utilice } \\
\text { accesorios eróticos para seducir }\end{array}$ \\
\hline
\end{tabular}

Pregunta sobre conductas sexuales violentas. Finalmente, se preguntó sobre conductas sexuales violentas realizadas durante el acto sexual sin consentimiento de la pareja, en un item con 9 propuestas (opciones de respuesta: Sí/No). La pregunta era: Durante una relación sexual consensuada, sin preguntarle a la otra persona si le gustaba, has... Con las siguientes opciones de respuesta: jalado el cabello, pegado, amarrado, mordido fuertemente, asfixiado momentáneamente, tapado la boca, dado cachetadas, gritado, insultado.

\section{Procedimiento}

Para las entrevistas grupales los estudiantes fueron contactados a través de sus profesores para participar en un estudio amplio sobre Sexualidad en la Juventud. Firmaron un consentimiento informado, y sus intervenciones fueron grabadas, transcriptas y posteriormente codificadas en el software NVivo 11. Las respuestas fueron analizadas con el método de análisis temático de Braun y Clarke (2006). El contenido de la pornografia surgió como transversal dentro del tema amplio de "Conductas sexuales en la época actual", específicamente de la pregunta abierta realizada a los participantes: ¿Cómo han influenciado la tecnología y los medios de comunicación en la sexualidad?

Para la participación en la encuesta en línea, los estudiantes fueron contactados por correo electrónico para solicitar su colaboración voluntaria, previo a la aceptación del consentimiento informado. Se proporcionó un link de Google Drive para acceder a la encuesta, que permaneció activa entre noviembre de 2018 y marzo del 2019. Para el análisis de la información, se utilizó el programa SPSS versión 25 (IBM Corp., 2017).

\section{RESULTADOS}

\section{Entrevistas grupales}

Con relación a la pregunta ¿Cómo han influenciado la tecnología y los medios de comunicación en la sexualidad?, las respuestas evidenciaron varias consecuencias, entre ellas el tema de pornografia, del cual se encontraron en total 48 referencias en las conversaciones con los jóvenes. Se pudo observar que los hombres hablaban más de ello (33 referencias vs. 15 en total en 
mujeres) y más extensamente: de todas las respuestas dadas a la pregunta planteada, $14 \%$ se dedicó al tema en los grupos de hombres, mientras que en los de mujeres solo se le dedicó el 5.31\%.

Se encontró que los temas principales alrededor de los cuales giraban las respuestas eran: acceso, incitación y afectación, relacionados entre ellos como se ve en el siguiente mapa conceptual (figura 1).

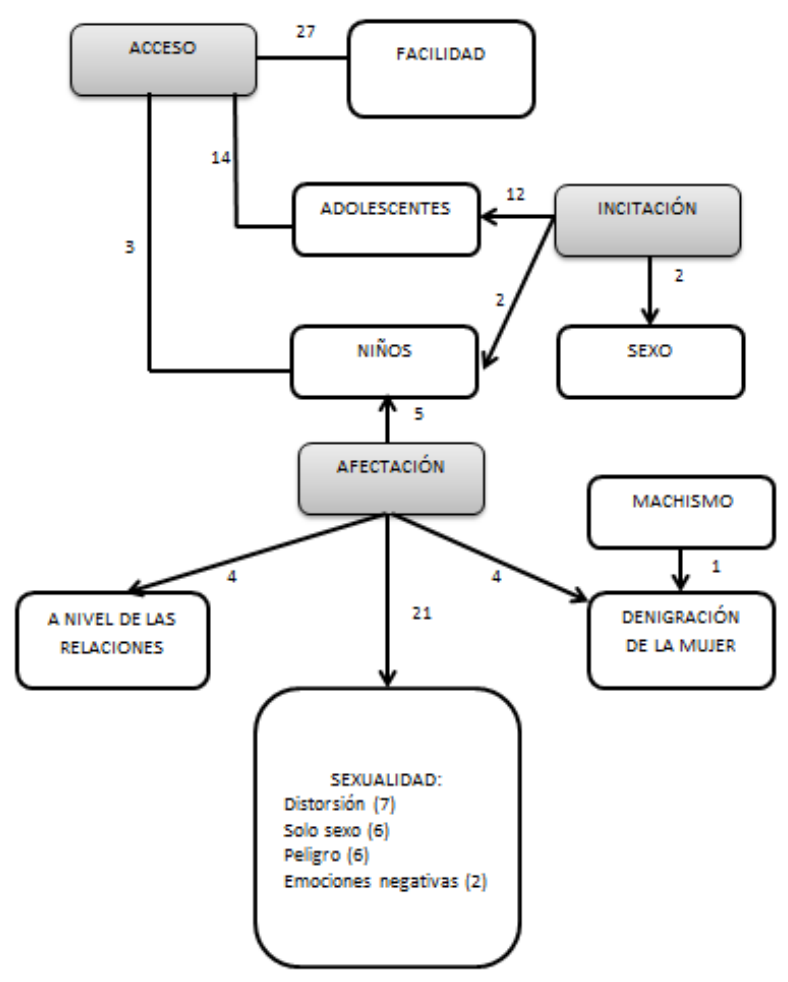

Figura 1. Mapa de relaciones del concepto pornografía. El número corresponde a las veces que se relacionaron entre sí los conceptos en las respuestas

Se puede ver que los participantes hablaron mucho de la facilidad de ACCESO a contenidos pornográficos ("Cualquiera que pueda usar Internet puede acceder a pornografia facilito", "Haces un clic y se te abre toda una ventana de anuncios"), y también del acceso por parte de adolescentes y, sobre todo, niños, a quienes ellos piensan que esto afecta. Los jóvenes también piensan que la pornografia INCITA/ESTIMULA a los niños y a los adolescentes incrementando su curiosidad y motivando a un despertar temprano. Y en general que provoca a buscar relaciones sexuales ("Hace que cualquier cantidad de personas se vuelquen a querer realizar el acto sexual"). La AFECTACIÓN no ocurre únicamente en los niños, puesto que se evidencian respuestas de consecuencias negativas de la pornografia sobre todo a nivel de la sexualidad: distorsiones ("Puede distorsionar las dimensiones del sexo, nos crea como un objetivo de disfrutarlo como se lo está proyectando"), de enfocar la sexualidad solo al sexo ("La sexualidad en el sentido vulgar"), es peligrosa para la sexualidad ("Puede llegar a degenerar o enfermar la mente" , "El primo de mi primo era muy jovencito y empezó a ser adicto a la pornografía y ya no podía dejar, creo que eso comenzó simplemente por las ganas de conocerse"), y que genera emociones negativas ("Frustración sexual", "Egoísmo"). Por otra parte, la pornografia afecta las relaciones, ya que se genera un espacio donde 
se oprime y cosifica a la mujer: "Yo estaba en un colegio masculino y era como que ahí todos veíamos pornografía. Por lo tanto, nos criábamos muy machistas y en alguna relación que tuve me di cuenta de que sí, era machista por esa falta de convivencia con mujeres, pero por internet mientras chateaba yo sentía que lo sabía todo, pero en realidad no sabía nada".

\section{Resultados de las encuestas}

De los 590 jóvenes encuestados, 460 fueron expuestos a pornografia por lo menos una vez en su vida $(78 \%)$, a la edad promedio de 14.5 años $(D E=$ 2.9). Se observa que el rango de edad donde se concentra la mayoría de la muestra oscila entre los 12 y 18 años, edades que corresponden a la adolescencia. Reconociendo que existen diferencias al interior del grupo llamado adolescencia, se hizo un análisis por subetapas que revela que es en la adolescencia temprana, entre los 12 y 14 años, donde la mayor parte de la muestra tuvo su primer contacto con material pornográfico (tabla 4).

Tabla 4.

Porcentaje de personas expuestas a pornografía por edad y etapa del desarrollo.

\begin{tabular}{rcc}
\hline $\begin{array}{r}\text { Edad de la primera } \\
\text { exposición (años) }\end{array}$ & Etapa del desarrollo correspondiente & Porcentaje \\
\hline $0-9$ & Infancia & 3.0 \\
$10-11$ & Etapa prepuberal & 6.5 \\
$12-14$ & Adolescencia temprana & 38.7 \\
$15-16$ & Adolescencia intermedia & 27.6 \\
$17-18$ & Adolescencia tardía & 19.3 \\
$19-24$ & Juventud & 4.8 \\
\hline
\end{tabular}

Las fuentes más comunes para el acceso fueron: internet (94.6\%); vídeos (72.6\%); televisión por cable (43.7\%); redes sociales (33.7\%); televisión (22.6); revistas $(16.3 \%)$ y prensa escrita $(7 \%)$.

Con relación al consumo de pornografia en la actualidad, los resultados indican que, de los 590 jóvenes de la muestra, 263 (44.6\%) lo hacen. De ellos, el $58.9 \%$ son varones y el $41.1 \%$ mujeres. El tiempo que le dedican a esta actividad en promedio es de casi tres horas semanales $(M=2.93 ; D E=4.73)$.

En cuanto al tipo de pornografia a la que acceden, se encontró que algunos son más populares que otros, y que hay diferencias entre géneros (tabla 5).

Las diferencias por género son significativas en las preferencias de pornografia homosexual $(p<.001)$ en mujeres, y en el caso de la pornografia con contenidos de sexo con adolescentes $(p=.004)$ en hombres. 
Tabla 5.

Tipo de pornografía consumida según el género del espectador.

\begin{tabular}{lcccccc}
\hline & \multicolumn{2}{c}{ Hombre } & \multicolumn{2}{c}{ Mujer } & & \\
\cline { 2 - 5 } & \multicolumn{1}{c}{ Sí (\%) } & No (\%) & Sí (\%) & No (\%) & $\chi^{2}$ & $p$ \\
\cline { 2 - 6 } Heterosexual & 92.3 & 7.7 & 97.2 & 2.8 & 2.91 & .088 \\
Homosexual & 32.3 & 67.7 & 55.6 & 44.4 & 14.19 & $<.001$ \\
Grupal (más de dos personas) & 59.4 & 40.6 & 63.0 & 37.0 & 0.34 & .555 \\
Que implique sometimiento & 35.5 & 64.5 & 32.4 & 67.6 & 0.26 & .605 \\
Que implique dominio & 42.6 & 57.4 & 38.9 & 61.1 & 0.35 & .549 \\
Dibujos de contenido & 34.8 & 65.2 & 31.5 & 68.5 & 0.32 & .570 \\
pornográfico & & & & & & \\
Escenas de violación/ violencia & 13.5 & 86.5 & 7.4 & 92.6 & 2.44 & .118 \\
sexual & 26.5 & 73.5 & 16.7 & 83.3 & 3.50 & .061 \\
Incestuoso & 56.1 & 43.9 & 38.0 & 62.0 & 8.40 & .004 \\
Con adolescentes & &
\end{tabular}

Nota: Hombres = 155 casos; Mujeres = 108 casos; $\chi^{2}=$ Chi-cuadrado; $p=$ significancia estadística

Para poder medir el posible impacto que tiene la pornografia en las actitudes hacia la pornografia, se realizaron comparaciones en el grupo de 460 personas que estuvieron expuestas alguna vez a pornografia, diferenciando aquellas que tuvieron una exposición puntual $(n=197)$ de las que dicen consumir actualmente pornografia $(n=293)$. Los resultados se muestran en la tabla 6.

Tabla 6.

Impacto del consumo de pornografía en las actitudes hacia la pornografía.

\begin{tabular}{|c|c|c|c|c|c|c|c|c|}
\hline \multirow{2}{*}{$\begin{array}{l}\text { Afirmaciones sobre la } \\
\text { pornografía }\end{array}$} & \multicolumn{2}{|c|}{$\begin{array}{l}\text { Personas que } \\
\text { ven pornografía }\end{array}$} & \multicolumn{2}{|c|}{$\begin{array}{l}\text { Personas que no } \\
\text { ven pornografía }\end{array}$} & \multicolumn{2}{|c|}{ Contraste } & \multirow[b]{2}{*}{$d$} & \\
\hline & M & DE & M & DE & $t$ & $p$ & & \\
\hline $\begin{array}{l}\text { Es parte de la vida sexual de la } \\
\text { persona }\end{array}$ & 3.67 & 1.03 & 2.91 & 1.16 & 7.31 & $<.001$ & .69 & $V>N V$ \\
\hline $\begin{array}{l}\text { Es parte de la vida sexual de la } \\
\text { pareja }\end{array}$ & 3.21 & 0.96 & 2.6 & 1.08 & 6.42 & $<.001$ & .60 & $\mathrm{~V}>\mathrm{NV}$ \\
\hline $\begin{array}{l}\text { Puede mejorar la vida sexual de la } \\
\text { pareja }\end{array}$ & 3.28 & 0.92 & 2.75 & 1.12 & 5.55 & $<.001$ & .51 & $\mathrm{~V}>\mathrm{NV}$ \\
\hline $\begin{array}{l}\text { Enseña cosas interesantes sobre } \\
\text { las relaciones sexuales }\end{array}$ & 3.46 & 0.96 & 2.89 & 1.15 & 5.73 & $<.001$ & .54 & $\mathrm{~V}>\mathrm{NV}$ \\
\hline $\begin{array}{l}\text { Está centrada en el placer } \\
\text { únicamente }\end{array}$ & 3.52 & 1.08 & 3.19 & 1.25 & 3.08 & .002 & .28 & $\mathrm{~V}>\mathrm{NV}$ \\
\hline Representa la vida sexual real & 1.76 & 0.93 & 1.7 & 0.90 & 0.74 & .456 & & \\
\hline Defiende la diversidad sexual & 2.93 & 1.15 & 2.56 & 1.14 & 3.40 & .001 & .32 & $\mathrm{~V}>\mathrm{NV}$ \\
\hline Defiende la libertad sexual & 3.11 & 1.13 & 2.76 & 1.24 & 3.20 & .001 & .30 & $\mathrm{~V}>\mathrm{NV}$ \\
\hline $\begin{array}{l}\text { Es una buena fuente de } \\
\text { educación sexual }\end{array}$ & 2.14 & 1.13 & 1.83 & 0.99 & 3.12 & .002 & .30 & $\mathrm{~V}>\mathrm{NV}$ \\
\hline $\begin{array}{l}\text { Es divertida } \\
\text { Debería ser objeto de }\end{array}$ & 3.58 & 0.96 & 2.73 & 1.13 & 8.68 & $<.001$ & .80 & $\mathrm{~V}>\mathrm{NV}$ \\
\hline $\begin{array}{l}\text { regulaciones estrictas o incluso } \\
\text { prohibida }\end{array}$ & 2.62 & 1.15 & 3.02 & 0.08 & -3.65 & $<.001$ & .49 & $\mathrm{~V}<\mathrm{NV}$ \\
\hline
\end{tabular}

Nota: Personas que ven (V): 263; Personas que no ven (NV): 197; p: Significancia; d: Prueba de tamaño del efecto de Cohen

Los resultados muestran que, en la mayoría de las afirmaciones propuestas, la percepción de los usuarios de pornografia difiere de la de los no usuarios. Así, solamente concuerdan en que la pornografia no representa la vida sexual real. En todas las otras afirmaciones, se observa un efecto de la exposición. Este es grande para la consideración de que la pornografía es divertida $(d=.8)$, mientras que se encuentra como moderado $(d>.5)$ para 
aspectos como: formar parte de la vida sexual personal o de la pareja, mejorar la vida sexual de la pareja, y enseñar cosas interesantes. Se observa también un pequeño efecto $(d>2)$ para las afirmaciones sobre la pornografia en relación con centrarse en el placer, defender la libertad y la diversidad sexual, o ser una buena fuente de educación. En todas estas afirmaciones, las personas que ven pornografia están más de acuerdo con ellas, mientras que aquellas que no la ven mantienen posturas más moderadas o en contra. Para la afirmación sobre la posible regulación/prohibición de la pornografia, el efecto es moderado en las personas que consumen pornografia $(d=.49)$, mientras que las personas que no ven pornografia son un poco más favorables con la idea de regularla o prohibirla.

Con la misma muestra $(n=460)$ se realizó un análisis para explorar si usar pornografia puede afectar otro tipo de actitudes, como por ejemplo aquellas sobre lo que se piensa respecto a los comportamientos o las relaciones sexuales y el placer. Se decidió hacer el análisis del impacto a nivel de items en lugar de las escalas generales, ya que las escalas usadas son preliminares, realizadas por los investigadores sobre la base de la información de las entrevistas grupales y a la literatura revisada, e interesaba ver el peso de los elementos. Los resultados muestran que la afectación se da en cinco items de la escala de actitudes hacia los comportamientos sexuales y en cuatro ítems de la escala de relaciones sexuales y placer (tabla 7 ).

Tabla 7.

Ítems pertenecientes a las escalas de Actitudes hacia los Comportamientos, y Relaciones sexuales y placer en donde se observa el impacto del consumo de pornografía.

\begin{tabular}{|c|c|c|c|c|c|c|c|c|c|}
\hline \multirow{2}{*}{$\begin{array}{l}\text { Escala de la que } \\
\text { proviene el ítem }\end{array}$} & \multirow[t]{2}{*}{ Afirmaciones } & \multicolumn{2}{|c|}{$\begin{array}{l}\text { Personas que ven } \\
\text { pornografía }\end{array}$} & \multicolumn{2}{|c|}{$\begin{array}{l}\text { Personas que no } \\
\text { ven pornografía }\end{array}$} & \multirow[b]{2}{*}{$t$} & \multirow[b]{2}{*}{$p$} & \multirow[b]{2}{*}{$d$} & \\
\hline & & $M$ & $D E$ & $M$ & $D E$ & & & & \\
\hline \multirow{5}{*}{$\begin{array}{l}\text { Comportamientos } \\
\text { sexuales }\end{array}$} & $\begin{array}{l}\text { Es normal jalar el cabello en } \\
\text { una relación sexual }\end{array}$ & 3.26 & 0.87 & 3.02 & 0.90 & 2.90 & .004 & .27 & $\mathrm{~V}>\mathrm{NV}$ \\
\hline & $\begin{array}{l}\text { Es normal decir cosas } \\
\text { obscenas en una relación } \\
\text { sexual }\end{array}$ & 3.12 & 0.91 & 2.87 & 1.01 & 2.72 & .007 & .26 & $\mathrm{~V}>\mathrm{NV}$ \\
\hline & $\begin{array}{l}\text { Es normal dar nalgadas en } \\
\text { una relación sexual }\end{array}$ & 3.55 & 0.90 & 3.30 & 0.90 & 2.89 & .004 & .28 & $\mathrm{~V}>\mathrm{NV}$ \\
\hline & $\begin{array}{l}\text { Es importante que la mujer } \\
\text { utilice accesorios eróticos } \\
\text { para seducir }\end{array}$ & 2.29 & 1.00 & 2.04 & 0.94 & 2.70 & .007 & .26 & $\mathrm{~V}>\mathrm{NV}$ \\
\hline & $\begin{array}{l}\text { Es importante que el } \\
\text { hombre utilice accesorios } \\
\text { eróticos para seducir }\end{array}$ & 2.23 & 0.94 & 2.01 & 0.92 & 2.57 & .010 & .24 & $\mathrm{~V}>\mathrm{NV}$ \\
\hline \multirow{4}{*}{$\begin{array}{l}\text { Relaciones } \\
\text { sexuales y placer }\end{array}$} & $\begin{array}{l}\text { Es excitante que el varón } \\
\text { domine y controle la relación } \\
\text { sexual y que la mujer se } \\
\text { someta }\end{array}$ & 3.05 & 1.17 & 2.77 & 1.19 & 2.48 & .013 & .24 & $\mathrm{~V}>\mathrm{NV}$ \\
\hline & $\begin{array}{l}\text { Es excitante que la mujer } \\
\text { domine y controle la relación } \\
\text { sexual y que el hombre se } \\
\text { someta }\end{array}$ & 3.07 & 1.16 & 2.77 & 1.11 & 2.80 & .005 & .26 & $\mathrm{~V}>\mathrm{NV}$ \\
\hline & $\begin{array}{l}\text { Me fijo en que mi pareja } \\
\text { disfrute cuando estamos } \\
\text { teniendo relaciones }\end{array}$ & 4.19 & 0.94 & 3.90 & 1.03 & 3.05 & .002 & .30 & $\mathrm{~V}>\mathrm{NV}$ \\
\hline & $\begin{array}{l}\text { Todos los hombres pueden } \\
\text { tener erecciones } \\
\text { prolongadas }\end{array}$ & 2.45 & 1.05 & 2.74 & 1.09 & -2.80 & .005 & .27 & $\mathrm{~V}<\mathrm{NV}$ \\
\hline
\end{tabular}

Nota: Personas que ven $=263 ;$ Personas que no ven $=197 ; p=$ significancia estadística; $d=$ Prueba de tamaño del efecto de Cohen 
En general, se observa que las personas que miran pornografia tienen actitudes más favorables que las que no ven pornografia hacia la posibilidad de realizar comportamientos como: jalar el cabello, dar nalgadas, fijarse en el placer de las parejas durante las relaciones sexuales. Para otras conductas, por ejemplo, el decir cosas obscenas durante la relación sexual y el opinar que las conductas de dominación en ambos sexos son excitantes, las personas que ven pornografia están medianamente de acuerdo, mientras que las que no la ven están más cercanas al desacuerdo. Con relación al uso de accesorios para seducir, aunque ambos géneros están en desacuerdo sobre la importancia del tema en ambos casos, el desacuerdo es mayor entre los no usuarios. Los análisis de magnitud de la diferencia entre grupos muestran que en todos los casos el efecto es bajo $(d<3)$.

Finalmente, se indagó el impacto que puede tener la pornografia en las conductas sexuales de las personas durante una relación sexual. Para ello, se preguntó a los jóvenes si durante una relación sexual habian realizado una serie de conductas sin el permiso de sus parejas, y se analizó el tema en función del ser usuario o no de pornografia. El análisis de diferencias muestra que las personas que ven pornografia difieren de manera significativamente superior de las que no lo hacen en el uso de las siguientes conductas ( $p>.05)$ : jalar el cabello, tapar la boca, dar cachetadas, asfixiar momentáneamente y agredir fisicamente a la pareja (tabla 8).

Tabla 8.

Impacto del consumo de pornografía en la ejecución de conductas sexuales no autorizadas durante una relación sexual.

\begin{tabular}{lcccccc}
\hline & \multicolumn{2}{c}{$\begin{array}{c}\text { Personas que ven } \\
\text { pornografía }\end{array}$} & \multicolumn{2}{c}{$\begin{array}{c}\text { Personas que no } \\
\text { ven pornografía }\end{array}$} & \\
\cline { 2 - 5 } Conducta & Sí (\%) & No (\%) & Sí (\%) & No (\%) & $\chi^{2}$ & p \\
\hline Morder fuertemente & 46.0 & 54.0 & 39.6 & 60.4 & 1.88 & .169 \\
Jalar el cabello & 45.6 & 54.4 & 30.5 & 69.5 & 10.88 & .001 \\
Tapar la boca & 27.8 & 72.2 & 16.8 & 83.2 & 7.69 & .006 \\
Pegar & 21.3 & 78.7 & 13.7 & 86.3 & 4.38 & .036 \\
Gritar a la persona & 16.3 & 83.7 & 10.7 & 89.3 & 3.04 & .081 \\
Dar cachetadas & 14.4 & 85.6 & 6.1 & 93.9 & 8.12 & .004 \\
Asfixiar & 10.6 & 89.4 & 4.1 & 95.9 & 6.77 & .009 \\
momentáneamente & 6.8 & 93.2 & 7.6 & 92.4 & 0.10 & .751 \\
Amarrar & 8.4 & 91.6 & 5.6 & 94.4 & 1.30 & .253 \\
Insultar & &
\end{tabular}

Nota: Personas que ven $=263 ;$ Personas que no ven $=197 ; p=$ significancia estadística

\section{COMENTARIOS}

El objetivo de esta investigación fue recabar información en población juvenil sobre la exposición a pornografia, su consumo y las consecuencias que esta tendría a nivel de las actitudes y conductas de los jóvenes.

Si bien individuos de todas las edades consumen pornografia, la edad de acceso a este contenido determina patrones de conducta específicos y afectaciones particulares (Baumel, Silva, Guerra, Garcia, \& Trindade, 2019; Castro-Calvo et al., 2017). En este contexto, Bischmann et al. (2017) indican que edades de exposición temprana a pornografia se asocian a la adherencia a normas de poder sobre la mujer o misoginia (vs. exposiciones más tardías a 
normas masculinas de "donjuanismo"). El estudio de Tardif et al. (2015) refiere así mismo que en adolescentes infractores sexuales se encontró que estos fueron expuestos a pornografia en edades tempranas. Esto es preocupante, por cuanto los resultados evidenciaron que, sin querer o buscándolo activamente, el $78 \%$ de los encuestados fueron expuestos a pornografia por lo menos una vez en su vida, en edades situadas mayoritariamente en la adolescencia temprana (12-14 años) e intermedia (15-16 años) y el $9.5 \%$ de ellos fue expuesto antes de los 11 años, siendo internet el mayor "responsable" de ello. Los hallazgos en cuanto edad de acceso son similares a los de otros estudios; así, Ballester et al. (2019) encuentran que la mitad de los adolescentes entre 14 y 17 años ven con regularidad pornografia en internet, y citan un estudio de la universidad de Indiana que sitúa la edad de acceso a los 13 años para chicos y a los 14 para chicas.

Es pertinente aquí recordar que la época de la adolescencia es particularmente importante para la sexualidad, tanto por factores específicos del desarrollo puberal como por otros que se entrelazan: aceptación de imagen corporal, descubrimiento de necesidades sexuales, desarrollo de la personalidad, aprendizaje de las relaciones sexuales y establecimiento de un sistema propio de valores sexuales (Garcia-Vega, Menéndez, Fernández, \& Cuesta, 2012).

Una parte considerable de los jóvenes encuestados no solamente fue expuesta a pornografia, sino que accede a ella con regularidad: el consumo bordea en promedio las 3 horas semanales, siendo entre 1 a 2 horas lo usual. E1 $44,7 \%$ de la muestra consume pornografia en la actualidad, un porcentaje inferior al hallado en otros estudios con la misma franja etaria (Nebot-García, Elipe-Miravet, Garcia-Barba, Antelo-Garcia, \& Ballester-Arnal, 2019; Rodríguez \& Santos, 2018; Villena \& Chiclana, 2018). El usuario es con mayor frecuencia un varón (59\%), hallazgo similar al de otros estudios en el tema (Ballester et al., 2019; Esteban, 2017; Nebot-García et al., 2019); esto puede ser porque es avalado socialmente que los hombres lo hagan, y hay un mayor tabú en temas de sexualidad para las mujeres. En todo caso, el consumo de este material suele ser bajo en mujeres (Matzumura \& Gutiérrez, 2015; Pulido, Gayoso, Pérez, \& Ruiz, 2015).

El género es algo que atraviesa también las actitudes de los usuarios de pornografia, como lo muestran los estudios. En hombres, existe un mayor grado de adicción percibido, mayor compulsión y esfuerzos para acceder a pornografia (Gomes, Fernandes, Ribeiro y Cardoso, 2019), mayor tiempo de consumo (Ballester et al., 2019), mayor aceptación de la pornografia (Escalante \& Torrico, 2016) y consumo por razones particulares, como el suprimir una carencia o escapar de relaciones insatisfactorias. Sin embargo, estas razones no se encuentran en las mujeres (Baumel et al., 2019). En el presente estudio se exploró específicamente la relación del género con el tipo de pornografia que se consume; las diferencias en preferencia por género en pornografia encontradas se ubicaban a nivel del sexo homosexual en mujeres y del sexo con adolescentes en varones.

Por una parte, el sexo homosexual puede no ser tan popular entre varones por los sesgos de género, que "penalizan" en la masculinidad al varón si fantasea con ello; esto está menos arraigado en las mujeres. Esta tendencia de preferencias no es particular de este estudio, puesto que los resultados a nivel global indican que las mujeres lo que más buscan y ven es pornografia lésbica, no así la pornografia gay en hombres que no aparece en ningún lugar 
entre las preferencias de hombres (Pornhub, 2018). Estas diferencias entre la sexualidad masculina y femenina han sido reportadas en otros estudios, que encontraron que las mujeres son "más maleables" en su sexualidad, lo que se conoce como plasticidad erótica (Peplau, 2003). Por otro lado, la preferencia mayor en varones por el sexo con adolescentes (vs. las mujeres) podría interpretarse como un asunto cultural, puesto que es socialmente "normal" que una pareja se constituya por un varón mayor y una mujer menor. Sin embargo, al observar los datos proporcionados por Pornhub (2018), se ve que la tendencia es similar a nivel mundial: así, la búsqueda de la palabra "teen" (adolescente) y el acceso a vídeos con adolescentes en la pornografia se encuentran en $7^{\circ}$ y $8^{\circ}$ lugar de las preferencias de varones, y no aparece en las de mujeres. Desde la psicología evolucionista se da una explicación que justifica la preferencia de los hombres por mujeres jóvenes, al plantear que existe evidencia transcultural de que las encuentran más atractivas por temas de fertilidad (Buss \& Schmith, 2011). Entre los rasgos que evidencian juventud y atraen a los hombres, están la "piel clara, suave, adiposidad facial, pelo brillante" (Buss \& Schmith, 2011, p. 776). Sin embargo, parece poco probable que la explicación evolucionista esté detrás de la búsqueda de pornografia con adolescentes justamente porque esta pornografia escoge actrices jóvenes con características sexuales que van en contra del concepto de "fertilidad", como caderas estrechas o senos pequeños. Empero, la tendencia es mundial: la búsqueda de vídeos de sexo con adolescentes es más popular en los hombres ( $7^{\circ}$ lugar), y se encuentra que las palabras "step mom" (madrastra) y "milf" (siglas para "mothers I like to fuck") están también entre las más buscadas en ambos géneros a nivel mundial (en mujeres: "madrasta" en $9^{\circ}$ lugar y "milf" en $10^{\circ}$; en hombres: "milf" en $2^{\circ}$ lugar y "madrastra" en $5^{\circ}$ ) (Pornhub, 2018). Al respecto, debemos reflexionar sobre lo ya subrayado por Szil (2018), que es la tendencia de la pornografia a popularizar y vender ciertas prácticas sexuales en la red y otros espacios, moldeando progresivamente las preferencias sexuales de las personas, fenómeno también señalado por Alario (2018). Esto gradualmente 'normaliza' ciertas prácticas, lo que en el caso específico del incesto y del sexo con adolescentes puede resultar peligroso. Así, Alario (2018) responsabiliza a los vídeos que erotizan el contacto con menores de las tasas de abuso sexual que se observan, porque "alimentan el deseo sexual masculino de abusar de niñas" (p. 73). En cuanto al uso del sexo incestuoso en la pornografia, Taylor (2011) denuncia que se está pornificando las violaciones intrafamiliares, erotizando al término 'incesto'.

Se observa con mucha preocupación las posibles consecuencias negativas de la pornografia, preocupación compartida por los jóvenes, como lo expresaron en las entrevistas grupales realizadas. Los jóvenes miran la posible afectación a nivel de las relaciones de pareja, de la sexualidad, de las conductas sexuales y de la degradación de la imagen de la mujer.

Los resultados de la encuesta realizada muestran efectos claros a nivel de las actitudes tanto hacia la pornografia como hacia el placer y las relaciones sexuales en personas que ven pornografia. Así, en primera instancia, las actitudes hacia la pornografia son más favorables cuando se ve pornografia que cuando no se ve. Por el contrario, cuando no se ve, se considera más su prohibición. Existen dos explicaciones posibles a los resultados encontrados: las personas que no están de acuerdo consideran más negativa a la pornografia y por eso no la ven; o la exposición a la pornografia "ablanda" la percepción de las personas frente a la misma. 
En segundo lugar, se exploró la posible modificación actitudinal en cuanto a la percepción sobre cómo tienen que darse las relaciones sexuales y el placer. Los resultados mostraron modificaciones en las personas que ven pornografia en actitudes más favorables hacia conductas violentas como jalar el cabello, dar nalgadas, decir cosas obscenas, conductas de dominación tanto en hombres como en mujeres. Esto puede deberse a que actualmente se popularizó en la pornografia y en películas el sado masoquismo (Cf: 50 sombras de Grey). Por tanto, es común que se observen en estas fuentes una asociación entre sexo y violencia.

El uso de accesorios para seducir también es común en la pornografia, y se ven en los resultados que, si bien hay desacuerdo en ambos géneros con esto, la oposición es menor en las personas que ven pornografia. Es interesante observar también que las personas que ven pornografia están más pendientes de las manifestaciones de placer, lo que puede ser explicado porque la pornografía es algo muy visual y se necesita mayor estímulo. Sin embargo, el consumo de pornografia no ha alterado algunas actitudes que fueron medidas y en las que no se observó diferencias entre usuarios y no usuarios, y que contemplaban los temas sobre todo de: distorsiones sobre las relaciones sexuales, estereotipos sobre la mujer y el hombre, y sobre el placer en ambos géneros y sus aspectos sociales, lo que podria corresponder a la conciencia que se evidenció también en las entrevistas efectuadas de la probable afectación en estos temas.

Finalmente, en cuanto a la posible afectación a nivel conductual, se evidenció que la frecuencia de conductas violentas durante el acto sexual es significativamente más elevada en los usuarios de pornografia. Así, las personas que ven pornografia en la actualidad en nuestra muestra, durante una relación sexual y sin autorización de su pareja, le han jalado el cabello, tapado la boca, dado cachetadas, asfixiado momentáneamente y pegado. Se observa una "normalización" de conductas violentas en las personas que consumen pornografia.

No se encontraron referencias en otros estudios que relacionen el consumo de pornografia con cambios conductuales similares a nivel sexual en población general, aunque existen algunos que sí relacionan la pornografia con cambios actitudinales en temas como predisposición a estilos sexuales agresivos (Ballester et al., 2019; Kjellgren et al, 2010; Del Barrio-Álvarez \& Garrosa, 2015; Wright, Tokunaga y Kraus, 2016; Ybarra et al., 2011) y creencias sexuales adversas, aceptación del mito de la violación y conservadurismo sexual (Emmers-Sommer, 2018).

Al ser imposible luchar contra el fenómeno de la difusión de pornografia en las redes y hasta en espacios cotidianos - la llamada "pornificación de la cultura" (Favaro \& De Miguel, 2016) - debemos retomar lo que los propios jóvenes propusieron en las entrevistas realizadas y con lo que concuerdan algunos autores: es esencial que exista una mediación entre la propuesta "cultural" pornificada de la sexualidad y el adolescente/joven que construye una noción de sexualidad.

No se trata de prohibir o restringir el acceso a la información en la Red, como un intento de "evitar" que se acceda a la pornografia, dado que esta se ha instaurado como agente pedagógico en nuestra sociedad (Rodríguez et al., 2018), debido a la ausencia de otros canales educativos en el tema. Se trata más bien de educar a los adolescentes y jóvenes para generar conciencia sobre lo que la pornografia realmente es: la quinta industria que más dinero mueve 
en el mundo (Rankia, 2017 ) y una red de prostitución para los que participan en ella (Szil, 2018; Tyler, 2015), contraponiéndola con lo que realmente es la sexualidad humana: "una dimensión fundamental del hecho de ser un humano: basada en el sexo, incluye al género, las identidades de sexo y género, la orientación sexual, el erotismo, la vinculación afectiva y el amor, y la reproducción" (Arango, 2008, p.5).

Finalmente, se concluye que la guía sobre la sexualidad debería ser dada por un lado por los padres, ya que el rol de la familia es subrayado como un factor protector frente al consumo de pornografia (Rivera, Santos, Cabrera, \& Docal, 2015). Por otro lado, reposa también en la educación formal la responsabilidad de educar en materia de identidad y de placer sexual (Rojo, 2018). Es de suma importancia que se pueda enfocar el tema justamente desde el marco de una educación sexual integral, con información clara que no involucre únicamente la genitalidad, sino otros aspectos como el placer, la afectividad, el énfasis en el respeto y la importancia del consenso en la sexualidad.

Uno de los límites de la presente investigación consiste en que el tema fue investigado dentro de un gran estudio de sexualidad, lo que hizo que no se profundizara en otros factores que pueden ser de interés, como el impacto en la construcción de la masculinidad en varones o de la concepción de lo femenino. Futuras investigaciones en el tema deberian además de realizarse con adolescentes, ya que este estudio evidencia que es en esa etapa en donde existen las primeras exposiciones a pornografia, y en donde también se deberian focalizar las intervenciones.

\section{REFERENCIAS}

Alario, M. (2018). La influencia del imaginario de la pornografia hegemónica en la construcción del deseo sexual masculino prostituyente: un análisis de la demanda de prostitución. Asparkía, 33 (4), 61-76. https://doi.org/10.6035/Asparkia.2018.33.4

Albury, K. (2014). Porn and sex education, porn as sex education. Porn Studies, 1(1-2), 172-181. https://doi.org/10.1080/23268743.2013.863654

Arango, I. (2008). Sexualidad humana. México: Manual Moderno.

Ballester, L., Orte, C., \& Pozo, R. (2019). Nueva pornografia y cambios en las relaciones interpersonales de adolescentes y jóvenes. En C. Orte, L. Ballester, \& R. Pozo, (Eds). Vulnerabilidad y resistencia: experiencias investigadoras en comercio sexual y prostitución (pp. 249-284). España: Universitat de les Illes Balears.

Baumel, C., Silva, P., Guerra, V., Garcia, A., \& Trindade, Z. (2019). Atitudes de jovens frente à pornografia e suas consequências. Psico-USF, 24(1), 131-144. https://doi.org/10.1590/1413-82712019240111

Bischmann, A., Richardson, C., Diener, J., Gullickson, M., Davidson, M. \& Gervais, S. (2017). Age and experience of first exposure to pornography, relations to masculine norms. Poster presented at the annual American Psychological Association convention.

Braun, V. \& Clarke, V. (2006). Using thematic analysis in psychology. Qualitative research in psychology, 3, 77-101. https://doi.org/10.1191/1478088706qp063oa

Buchholtz, K. (11 de febrero 2019). Chart: How much of the internet consists of porn? Statista. Recuperado de: https://www.statista.com/chart/16959/share-of-the-internet-that-is-porn/

Buss, D. \& Schmitt, D. (2011). Evolutionary psychology and feminism Sex Roles, 64, 768-787. https://doi.org/10.1007/s11199-011-9987-3

Castellanos (2006). Erotismo, violencia y género. Deseo femenino, femineidad y masculinidad en la pornografia. La manzana de la discordia, 1(2), 53-65. https://doi.org/10.25100/lmd.v1i2.1423

Castro-Calvo, J., Ballester-Arnal, R., Giménez-García, C., \& Gil-Juliá, B. (2017). Comportamiento sexual online en adultos mayores. International Journal of Developmental and Educational Psychology, 2 (1), 89-97. https://doi.org/10.17060/ijodaep.2017.n2.v2.1082

Del Alcázar, J. (2018). Ecuador Estado Digital Oct/18. Formación General Bussiness Advisors. Recuperado de: https://drive.google.com/file/d/116eZRcn-FH-cLVWmGGlt3jAn_SdG1aTL/view

Del Barrio-Âlvarez, E. \& Garrosa, E. (2015). ¿Educando en igualdad? Análisis de la triada pornografiadiscriminación-violencia. Feminidad y masculinidad en la pornografia convencional. Journal of Feminist, Gender and Women Studies, 1, 29-39

Emmers-Sommer, T. (2018). Reasons for pornography consumption: associations with gender, psychological and physical sexual satisfaction, and attitudinal impacts. Sexuality \& Culture, 22, 48-62. https://doi.org/10.1007/s12119-017-9452-8 
Escalante, C. \& Torrico, C. (2016). Actitudes hacia la pornografia en los estudiantes de la UPSA. Revista de estudiantes de Psicologia, 4, 12-15.

Esteban, A. (2017). El consumo de pornografia: diferencias de género e influencia en las relaciones de pareja (Tesis de grado). Madrid: Universidad Francisco de Vitoria.

Farvid, P. \& Braun, V. (2014). The "Sassy Woman" and the "Performing Man": Heterosexual casual sex advice and the (re)constitution of gendered subjectivities. Feminist Media Studies, 14(1), 118-134. https://doi.org/10.1080/14680777.2012.724027

Favaro, L. \& De Miguel, A. (2016) ¿ Pornografia feminista, pornografia antirracista y pornografia antiglobalización? Para una crítica del proceso de pornificación cultural. Labrys, Études Féministes/Estudos Feministas, 29, 1-20.

García-Vega, E. G., Menéndez, E. M., Fernández, P., \& Cuesta, M. (2012). Sexualidad, anticoncepción y conducta sexual de riesgo en adolescentes. International Journal of Psychological Research, 5(1), 79-87. https:// doi.org/10.21500/20112084.764

Gomes, A., Fernandes, A., Ribeiro, R., \& Cardoso, J. (2018). Ciberpornografia e atitudes sexuais em estudantes universitários: estúdio exploratório. Actas do $10^{\circ}$ Congreso Nacional de Psicologia da Saúde, Lisboa.

Harkness, E., Mullan, B., \& Blaszczynski, A. (2015). Association between pornography use and sexual risk behaviors in adult consumers: a systematic review. Cyberpsychology, Behavior, and Social Networking, 18 (2), 59-71. https://doi.org/10.1089/cyber.2014.0343

IBM Corp. (2017). IBM SPSS Statistics for Windows (Version 25.0). [software]. Armonk, NY: IBM Corp.

Johnson, J., Ezzell, M., Bridges, A. \& Sun, C. (2019). Pornography and heterosexual women's intimate experiences with a patner. Journal of Women's Health, 28(9), 1254-1265. https://doi.org/10.1089/jwh.2018.7006

Kjellgren, C., Priebe, G., Svedin, C. \& Langstrom, N. (2010). Sexually coercive behavior in male youth: Population survey of general and specific risk factors. Archives of Sexual Behavior, 39, 1161-1169. https://doi.org/10.1007/s10508-009-9572-9

Matzumura, J. \& Gutiérrez, H. (2015). Conocimientos y comportamentos sexuales em estudiantes de obstetrícia de dos universidades de Lima durante el 2014. Revista centroamericana de obstetrícia y ginecologia, 21 (1), 8-14.

Montoya, K. \& Vélez, C. (2016). Concepciones de erotismo que tienen los jóvenes de la IEHAJ a partir de los relatos de su experiencia con la pornografia (Tesis de grado). Colombia: Universidad Católica de Pereira.

Nebot-García, J., Elipe-Miravet, M., Garcia-Barba, M., Antelo-García, A., \& Ballester-Arnal, R. (2019). Consumo de pornografia y malestar asociado: diferencias entre hombres y mujeres. Trabajo presentado en las V Jornadas de Investigación para el alumnado de la FCS de la Universitat Jaume I, Castellón, España. https://doi.org/10.6035/AgoraSalut.2020.7.24

Oddone-Paolucci, E., Genuis, M., \& Violato, C. (2017). A meta-analysis of the published research on the effects of pornography. En C. Violato \& E. Oddone-Paolucci (Eds.). The Changing Family and Child Development (pp. 48-59). London: Routledge. https://doi.org/10.4324/9781315201702-4

Peplau, L. A. (2003). Human sexuality: how do men and women differ?. Current Directions in Psychological Science, 12(2), 37-40. https://doi.org/10.1111/1467-8721.01221

Peter, J. \& Valkenburg, P. (2008). Adolescents' exposure to sexually explicit internet material, sexual uncertainty, and attitudes toward uncommitted sexual exploration: is there a link?. Communication Research, 35, 569-601. https://doi.org/10.1177/0093650208321754

Pornhub (2018). Pornhub insights: 2018 year in review. Recuperado de https://www.pornhub.com/insights /2018-year-in-review.

Pulido, M., Gayoso, J., Pérez, C., \& Ruiz, M. (2015). Algunas dimensiones de la experimentación sexual: una comparación por sexos. Revista electrónica de Psicología Iztacala, 18 (4), 1690-1705.

Rankia (2017). Top 11 ranking industrias que más dinero mueven en el mundo y cómo invertir en ellas. Recuperado de https://www.rankia.com/blog/bolsa-al-dia/3534358-top-11-ranking-industrias-quemas-dinero-mueven-mundo-como-invertir-ellas.

Real Academia Española. (2001). Diccionario de la lengua española (22. ${ }^{\mathrm{a} e d .) .}$ Recuperado de https://dle.rae.es/?id=ThYXkZ3

Rivera, R., Santos, D., Cabrera, V., \& Docal, M. (2015). Consumo de pornografia on-line y off-line en adolescentes colombianos. Comunicar. Revista científica de comunicación y educación, 46 (1), 37-45. https://doi.org/10.3916/C46-2016-04

Rodríguez, M. \& Santos, A. (2018). Consumo de pornografia en universitarios mexicanos y cómo lo viven. Revista Psicología sin Fronteras, 1 (2), 5-25.

Rodríguez, S., González, L., \& Paulini, S. (2018). Narrativas sobre los cuerpos de las mujeres: sexualidad, publicidad y pornografia. Ponencia en las XXII Jornadas Nacionales de Investigadores en Comunicación, San Salvador de Jujuy.

Rojo, I. (2018). Educando en cuerpos pornográficos: un análisis más allá de la excitación. Revista de Estudios Socioeducativos, https://doi.org/10.25267/Rev_estud_socioeducativos.2019.i7.07

Rothman, E., \& Adhia, A. (2016). Adolescent pornography use and dating violence among a sample of primarily black and hispanic, urban-residing, underage youth. Behavioral Science, 6(1), 1-11. https://doi.org/10.3390/bs6010001

Rothman, E., Kaczmarsky, C. , Burke, N., Jansen, E., \& Baughman, A. (2014): "Without porn ... I wouldn't know half the things I know now": a qualitative study of pornography use among a sample of urban, low-income, black and hispanic youth. The Journal of Sex Research, 52 (7), 736-746. https://doi.org/10.1080/00224499.2014.960908

Szil, P. (2018). En manos de hombres: pornografia, trata, prostitución. Atlánticas. Revista Internacional de 
Estudios Feministas, 3(1), 113-135. https://doi.org/10.17979/arief.2018.3.1.3081

Tallon-Hicks, Y. [2016]. Is the Porn Brain our new Sex Educator? [Video]. YouTube. https://www.youtube.com/watch?v=1xlGFtg4Z2c

Tardif, M., Pascuzzo, K., \& Costa, M. (2015). Adolescentes autores de abuso sexual: atitudes e comportamentos face à sexualidade. Adolescência e Saúde, 12 (3), 33-42.

Taylor, C. (2001). The pornification of rape. En Tankard, M. \& Bray, A. (Eds). Big Porn Inc. Exposing the harms of the global pornography industry. Australia: Spinifex Press Pty Ltd.

Tyler, M. (2011). Selling sex short: The pornographic and sexological construction of women's sexuality in the West. Newcastle, UK: Cambridge Scholars.

Tyler, M. (2015). Harms of production: theorising pornography as a form of prostitution. Women's studies international forum, 48, 114-123. https://doi.org/10.1016/j.wsif.2014.11.014

Velasco, A. \& Gil, V. (2017). La adicción a la pornografia: causas y consecuencias. Drugs and Addictive Behavior, 2 (1), 228-237. https://doi.org/10.21501/24631779.2265

Villena, A. \& Chiclana, C. (junio, 2018). Consecuencias del consumo de pornografia en las relaciones personales y sexuales. Ponencia en la IX Reunión Internacional de Sexualidad, Psiquiatría y Tecnologia, Salamanca.

Wright, P., Miezan, E., Sun, C., \& Steffen, N. (2019). Relational monogamy, condomless sex, and perceptions of pornography as sexual information in an English sample. Sex Health, 16 (1), 70-74. https://doi.org/10.1071/SH18050

Wright, P., Tokunaga, R., \& Kraus, A. (2016). A Meta-Analysis of Pornography Consumption and Actual Acts of Sexual Aggression in General Population Studies. Journal of Communication, 66(1), 183-205. https://doi.org/10.1111/jcom.12201

Ybarra, M., Mitchell, K., Hamburger, M., Diener-West, M. \& y Leaf, P. (2011). X-rated material and perpetration of sexually aggressive behavior among children and adolescents: Is there a link?. Aggressive Behavior, 37,1-18. https://doi.org/10.1002/ab.20367

Recibido 18-12-2020 | Aceptado 10-08-2020

Este trabajo se encuentra bajo una Licencia Creative Commons Atribución 4.0 Internacional que permite a terceros utilizar lo publicado siempre que se dé el crédito pertinente a los autores y a Psicodebate 